monilethrix. The family has never had, on either side, any relative engaged in farming. This $I$ ascertained in making inquiry to exclude the possibility of there being consanguineous relationship with the family reported by the late Professor Sir Thomas McCall Anderson in his Treatise on Diseases of the Skin (second edition, 1894). Nor is the family related to the group of twenty-two cases, including five generations, previously reported by me, of which all the adult males from the first generation had followed the occupation of coal-miner.

\section{Discussion and Conclusion}

Arresting features in this familial and congenital group are:

(a) The mother appears to have been the first member of the family to show signs of the disease. I have failed to get any history of the affection in previous generations, and hei brother and sister are unaffected.

(b) Associated keratosis pilaris tends, as demonstrated in her own case, to disappear with advancing years. To this feature of the disease I made allusion in my earlier report.

(c) Assuming that there was some slight inaccuracy in the mother's statement as to the age when improvement appeared in the case of her older boy, it would seem that in some cases the onset of puberty, a period of increased glandular activity, has a beneficial influence in stimulating the growth of the hair of the scalp; this is certainly shown in the case of a boy in the previous group.

(d) The disease in the girl sent to me by Dr. Howat on August 24th, 1933, had, previously to his having seen her, been diagnosed as tinea tonsurans. Reference to such a diagnostic error having been made was mentioned in my earlier article.

It appears to me exceptional to be able to report a second series of cases after so short an interval, for monilethrix is a rare affection. It is well, therefore, always to bear this condition in mind, especially when examining the scalps of children with respect to fitness for attendance at school.

\section{TREATMENT OF HAMMER-TOE \\ sY}

\section{W. SAYLE CREER, M.B., M.Ch.ОRTh. LIVERPOOL}

In treating this deformity, in either the congenital or the acquired form, several very troublesome complications have been met. They were due in every case to difficulties of splintage after the common operation of wedge excision of the proximal interphalangeal joint. An account of the complications and their causes, with a description of a new splint which eliminated them, may not be without interest to those who use this method of treatment.

\section{Operative Methods}

In a review of the subject Trethowan ${ }^{1}$ mentions four operative methods of curing hammer-toe. The first is to amputate the whole toe, which, while removing the deformity, is a very sweeping operation, though one has yet to learn of a more satisfactory treatment in the case of a deformed little toe, while amputation of the terminal phalanx of the other three smaller toes, if this is the only affected part, gives good results. The great disadvantage of amputation of the whole of any of these toes is that it leaves a gap which the two neighbouring toes attempt to fill in. The alteration of their axes not only adds fresh deformities, but may also give rise to further disability by the production of pain.

The second treatment is to excise the proximal phalanx. Although this filleting leaves a flail-toe, it is probable that very little disability would arise in these days when we seldom appear without the support of a shoe. The method does not, however, conform to the ideals of modern plastic and reconstructive surgery.

In the third method, tenotomy of the tendons and the joint capsule, we have an effective treatment in selected cases. As it is very important that the corrected position be retained until all fear of relapse is past, some form of splintage is necessary. I suggest that the new splint, described later, is the easiest and most comfortable that can be used.

Finally, we have the common operation of interphalangeal arthrodesis by wedge excision of the affected joint, which in the majority of cases will be the proximal joint. After this latter operation a perfect result will leave the proximal interphalangeal joint stiff in almost full extension, and the metatarso-phalangeal and distal joints free from any hyperextension. Such a toe can

${ }^{2}$ Trethowan, W. H.: Lancet, 1925, i, 1257. grip the ground like a normal toe, but must be painless and unable to re-deform. Experience has led many of us to believe that to fulfil these conditions the arthodesis must be bony, for a long fibrous ankylosis is easily strained, with consequent pain, and will not remain extended, while even a short fibrous union re-deforms as the months pass. Now in the full list of causes of failure of consolidation in fractures and arthrodeses there can be no doubt that considerable angular movement occupies a prominent place. In the toe the phalanges are so minute that we often fail to appreciate how much of this type of movement takes place when what appears to be only negligible motion is allowed at the ends distant from the arthrodesis. Its prevention requires an efficient form of splintage.

Use of Splintage

In the past two types of splintage have been used. Of the first, collodion and ribbon gauze, I have no experience in this connexion, but from observation of its use in other conditions-for example, fractures-for which it is excellent, I should be surprised to find it sufficiently strong to retain the corrected position, even if tenotomy is performed at the same time as the wedge excision. Its use also necessitates complete removal-not an entirely painless procedure-when the sutures are removed. I believe that removal of the splintage for even a short time at this period to be detrimental to the rapid and certain attainment of bony fusion.

The second type used is a small wooden or metal splint affixed by means of adhesive tape. Satisfactory fixation had real difficulties. Because of their shape it is no easy matter to attach any straight object either to the dorsal or to the plantar surface of the foot and toe. When applied on the plantar aspect the thick tread of the foot keeps the splint away from good contact with the proximal phalanx, and tends to hyperextend the excised joint. If attached on the dorsal surface, it plantar-flexes the metatarso-phalangeal joint to such an extent that the patient is in great pain for several days. Even with careful padding above and below the dressing over the wound it is difficult to avoid this, while at times the arthrodesis is found later to have been splinted in partial flexion.

The net result has often been a splint that " fits where it touches " without immobilizing, and touches where it should not fit, with resulting malposition. This touching, or rather pressing, where it should not is another source of considerable trouble, particularly when the splint is on the dorsal aspect. 'Despite careful padding the splint presses on the wound through the dressing, frequently causing moisture of the skin edges and delayed healing, 
and sometimes sloughing or even sepsis from ischaemia. This sloughing of itself decreases the possibility of bony fusion, and if the sepsis appears to be severe the surgeon may be induced to remove the splint, and therewith the immobilization while treating it. In any case, as with collodion-gauze, the splint has to be taken off while the sutures are removed, and it has been our experience that many are not reaffixed, because the patient refuses to endure the pain or for a variety of other reasons.

\section{An Improved Splint}

It was the dissatisfaction induced by these experiences that led to the search for a splint free from the inherent disabilities of a straight bar, and with the able assistance

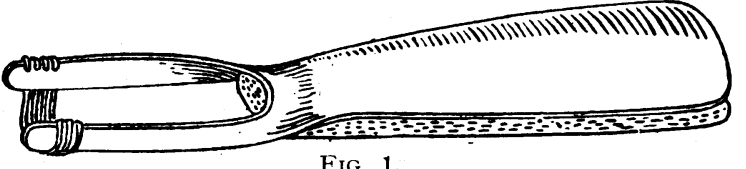

FIG. 1.

of the makers ${ }^{2}$ the one illustrated was devised (Fig. 1). It is constructed of duralumin of sufficient thickness to be rigid and yet mouldable by hand.

The expanded portion is curved from side to side to fit the dorsum of the foot, but is plane from the extremity to just short of the bifurcation, where it takes a slight upward curve corresponding to the shape of the foot at the metatarso-phalangeal joint. At this point the width of the splint is approximately that of the toe, and the side-to-side curvature becomes very pronounced, so that after the bifurcation the two " prongs " have their surfaces in parallel planes, and lie along each side of the toe.

The flat portion is lined prior to application with a quarter-inch-thick adhesive felt, and it is important that this should extend right up to the bifurcation. Strapping binds it snugly to the dorsum of the foot. The bifurcation should lie just proximal to the incision over the excised joint, in order that there shall be downward pressure over the proximal phalanx, but not over the wound (Fig. 2).

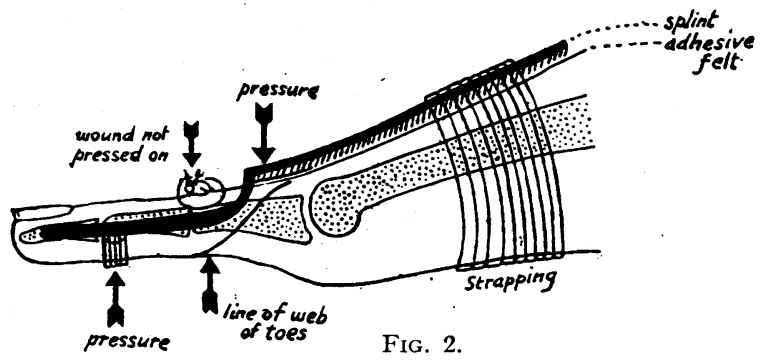

Thus there is prevention of dorsiflexion of the metatarsophalangeal joint, yet the wound is free from pressure, and can be inspected and dressed without need to touch the splint. The two prongs are adjusted for the width of the toe, and extend along the sides as far as the tip. Narrow strapping from one to the other passes beneath the head of the middle phalanx (not the terminal phalanx) and provides the essential pressure which keeps the arthrodesed joint extended. The prongs in themselves prevent lateral movement. A small amount of thin bandage wrapped round the whole keeps the dressing in place and completes the application.

The total strapping and bandage is so small that the splint has the advantage of occupying so little space that the patient can soon wear loose slippers and can safely hobble about, so relieving the tedium of the six or eight weeks of waiting for consolidation.

I offer my thanks to Mr. Watson Jones for his interest in the evolution of the splint and in its use on his patients at the Liverpool Royal Infirmary ; and to the instrument makers for preparing the splint to my designs and for supplying the illustration blocks.

${ }^{2}$ F. H. Critchley, Ltd., Liverpool, surgical instrument makers.

\section{Clinical Memoranda}

\section{Combined Intrauterine and Extrauterine Pregnancy}

This case is reported owing to its comparative rarity and the difficulties to which it gave rise in diagnosis.

Mrs. C., aged 28, 3-para, was admitted in a collapsed state to St. Mary's Hospital, Islington, on January 30th, 1934, as a case of threatened miscarriage. Her history was that she had been seized with sudden severe abdominal pain after two months' amenorrhoea. There had been a slight show a month before. On examination she was losing slightly, and the uterus was too large for the period of amenorrhoea. A provisional diagnosis of hydatidiform mole was made. Her condition improved rapidly after admission, but she continued to have a certain amount of abdominal pain. The bleeding ceased, and the Aschheim-Zondek test was positive. Her temperàture rose only very slightly above normal. There is a note that examination revealed a tender, soft swelling in the right fornix, pushing the uterus over to the left, and at a later date a note states that a mass, which could be seen to alter in consistency, was felt reaching to the umbilicus.

On February 20th I was asked to see her, with a view to examining her under an anaesthetic and proceeding to do a laparotomy if necessary. I found an ill-defined cystic mass in the pouch of Douglas, which I thought might be an infected ovarian cyst complicating pregnancy, and I decided to operate at once.

On opening the abdomen there was a large quantity of blood clot in the pouch of Douglas, caused by a right tubal abortion. The uterus was the size and consistency of a sixteen-weeks pregnancy. I removed the clot and the outer end of the right tube, and the pathological report on the specimen was: Tubal gestation; blood clot containing a few chorionic villi in lumen of and outside Fallopian tube.

The convalescence was uneventful, and she returned to hospital for her confinement, and gave birth to a healthy child on September 10th, 1934.

\section{COMMENTARY}

This condition is sufficiently rare to find no mention in certain standard textbooks of obstetrics. Curtis, ${ }^{i}$ however (1933), refers to it, and states that 243 cases were collected by Neugebauer in 1913, 276 were reported by Novak in 1926, and Stein, in 1928, brought the total up to 279 .

As might be expected, it is a dangerous condition for both mother and child. Stein ${ }^{2}$ gives the maternal mortality in a series of 202 cases as 21 per cent., and the intrauterine foetal mortality in 202 cases as 61 per cent. It is, however, very interesting to note that the extrauterine foetus seems to be far more likely to develop to term when accompanied by an intrauterine pregnancy than when present alone, and Stein, in a series of 164 cases, found that thirty-eight went to term and six survived. In a case reported by Novak $^{3}$ both intrauterine and extrauterine pregnancies developed to term, and the extrauterine child survived, while the intrauterine one died at birth. Novak, in his series, refers to a triplet pregnancy, the uterus containing twins, which survived.

I am indebted to Sir Frederick Menzies and to Dr. Turtle the medical superintendent of St. Mary's Hospital, Islington (L.C.C.), for permission to report this case.

\section{Margaret M. Basden, M.D.,} F.R.C.S., F.C.O.G.

Consulting Gynaecologist and Obstetrician, L.C.C. ; Surgeon, South London Hospital for Women

London, W. Obstetric Surgeon, Mothers' Hospital, Clapton.

\footnotetext{
Curtis: Obstetrics and Gynaecology.

${ }^{2}$ Amer. Journ. Obstet., 1928, xv.

Surg., Gynecol. and Obstet., 1926, lxiii.
} 